\title{
A Fast and Automatic Approach for Removing Artefacts due to Immobilisation Masks in X-ray CT
}

\author{
Mohammad Hashem Ryalat ${ }^{\S}$ Stephen Laycock $^{\S}$ Mark Fisher $^{\S}$
}

\begin{abstract}
Immobilisation masks are fixation devices that are used when administering radiotherapy treatment to patients with tumours affecting the head and neck. Radiotherapy planning X-ray Computer Tomography (CT) data sets for these patients are captured with the immobilisation mask fitted and manually editing the X-ray CT images to remove artefacts due to the mask is time consuming and error prone. This paper represents the first study that employs a fast and automatic approach to remove image artefacts due to masks in X-ray CT images without affecting pixel values representing tissue. Our algorithm uses a fractional order Darwinian particle swarm optimisation of Otsu's method combined with morphological post-processing to classify pixels belonging to the mask. The proposed approach is tested on five X-ray CT data sets and achieves an average specificity of $92.01 \%$ and sensitivity of $99.39 \%$. We also present results demonstrating the comparative speed-up obtained by fractional order Darwinian particle swarm optimisation.
\end{abstract}

Index Terms-Immobilisation Mask, CT Images, Head and Neck Cancer.

\section{INTRODUCTION}

Head and Neck Cancer (HNC) generally refers to a group of different malignant tumors that develop in or around the throat, larynx, nose, sinuses, and mouth [1]. It is the eighth most common cancer in the UK (2014), accounting for 3\% of all new cases [2] and for about 3\% of all cancers in the United States [3]. An estimated 61,760 people have developed HNC in the US within 2015 [4].

A course of Radiotherapy Treatment (RT) is typically prescribed for patients diagnosed with HNC. RT directs high energy ionising radiation to destroy malignant cells, but it must be accurately targeted to limit harm to healthy cells. A typical course of radiotherapy treatment for HNC is delivered in fractions over several weeks and masks (Figure 1) are employed to ensure the patient can be consistently repositioned for each dose fraction. X-ray CT data used for planning HNC radiotherapy treatment contain artefacts due to the mask (Figure 6(a)) which can make planning more difficult and can be troublesome if images of the skull are needed. This paper investigates an automatic approach for removing them.
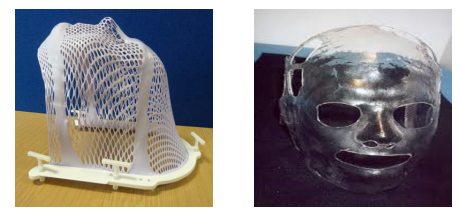

Fig. 1. Immobilisation masks (left) Thermoplastic (right) Polyethylene.

Since X-ray CT data of HNC are often acquired prior to RT many publicly available X-ray CT data sets include immobilisation masks. Artefacts within the X-ray CT due to immobilisation masks

$\S\{$ m.ryalat, s.laycock, mark.fisher\}@uea.ac.uk are with the School of Computing Sciences, University of East Anglia, Norwich, UK. are sometimes undesirable. Many segmentation of the brain, lateral ventricles, and skull are made more complicated by artefacts due to the mask. For example [5] and [6] need to render a reconstructed 3D CT volume of the head. Removing the mask by manually editing individual CT image slices, is time consuming and prone to errors particularly in the regions where the mask contacts the skin. Development of a robust approach to automatically remove the masks from the X-ray CT slices represents an appreciable saving in time and avoids the possibility committing manual errors.

There are numerous studies related to the segmentation of intra-cranial therapy relevant structures in X-ray CT images [7][10] but in our knowledge none address automated segmentation of immobilisation masks. Our automatic approach employs an exhaustive search (optimised by fractional order Darwinian particle swarm optimisation) for clusters of pixel values that satisfy Otsu's criteria [11] and are indicative of the classes 'immobilisation mask' and 'background'. Morphological image processing operations are then used to refine the labels and finally we remove the mask using a 'search and replace' strategy. Although we cannot draw direct comparisons for our values of specificity and sensitivity from previous published work we highlight other comparative benchmarks in section IV. The remainder of this paper is organised as follows. Section II presents the proposed approach, section III describes the datasets used for evaluation and presents results. Section IV provides a discussion and section V draws conclusions.

\section{Materials And Methods}

Five anonymised X-ray CT data sets have been used in this study. The first three data sets $(512 \times 512 \times 155,512 \times 512 \times 146$, $512 \times 512 \times 151$, helical, pixel-spacing $1.367 \times 1.367 \mathrm{~mm}$, slicethickness $2.5 \mathrm{~mm}$ ) were acquired at St James's University Hospital NHS Foundation Trust, Leeds, UK and the other two data sets $(512 \times 512 \times 130,512 \times 512 \times 156$, pixel-spacing $1.08 \times 1.08 \mathrm{~mm}$ $0.98 \times 0.98 \mathrm{~mm}$, slice-thickness $3.14 \mathrm{~mm}$ ) were downloaded from the Cancer Imaging Archive (TCIA)/Head-Neck-Cetuximab [12] [13]. The basic steps of the method are presented in Figure 2 and described below in part- $A$ to part- $C$.

Part-A

We begin with an X-ray CT image that includes an immobilisation mask and then use the Fractional Order Darwinian Particle Swarm Optimisation (FODPSO) algorithm [14] to return a segmented image by labelling pixels in six different classes under Otsu's criterion [11]. Section III explains that we found using six class labels leads to better results since all or most of the pixels belonging to the mask are in one class.

A heuristic search (Algorithm 1) processes the pixels of the labelled image starting from the top middle pixel until it finds the first pixel having a different label (see Figure 3(d)). We store 


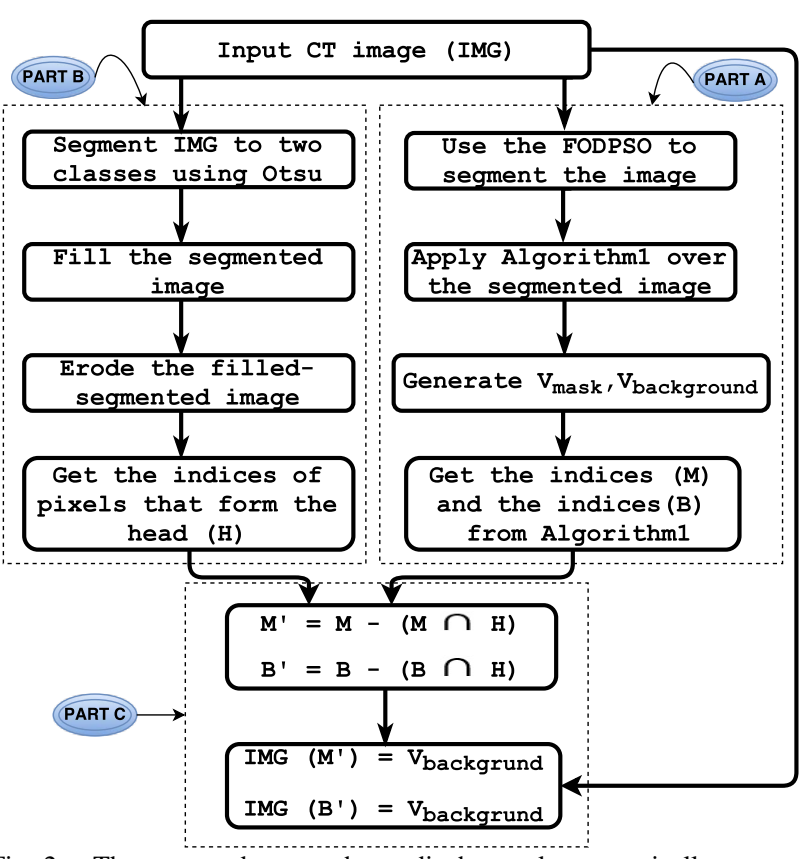

Fig. 2. The proposed approach to edit the mask automatically.

its index and value as [row_index, mid_column)], v_mask respectively. The value stored in $v \_$mask represents the immobilisation mask pixel label. The search algorithm assumes the pixel at [row_index - 1, mid_column)] is a background pixel label $v \_b g \_i n \_C T \_i m g$. By matching $v \_m a s k$ and $v \_b g \_i n \_C T \_i m g$ the search algorithm finds sets of indices $(\mathbf{M})$ and $(\mathbf{B})$ representing pixels labelled as 'mask' and 'background'. These sets ( $\mathbf{M}$ and $\mathbf{B}$ ) are further refined in the steps described in part-B.

Algorithm 1 Finding the pixels that represent the immobilisation mask and those that represent the background pixels

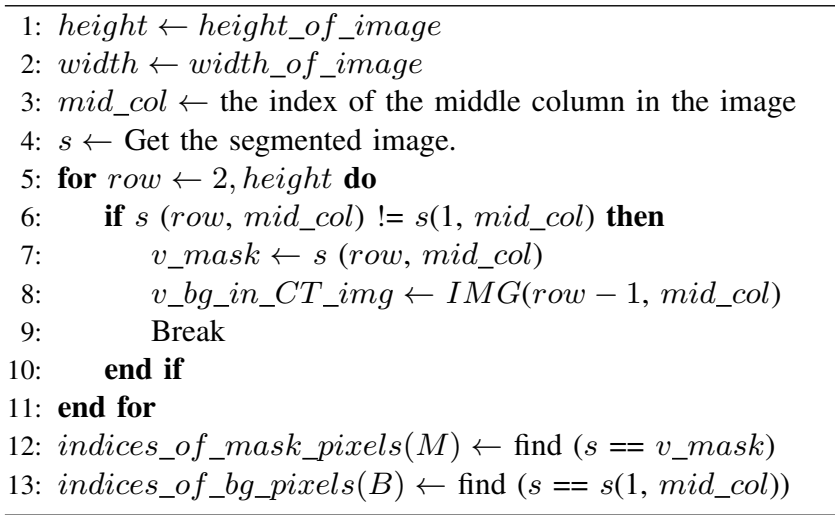

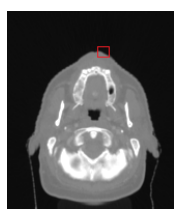

(a)

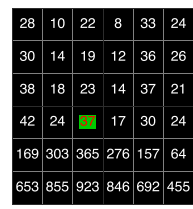

(b)

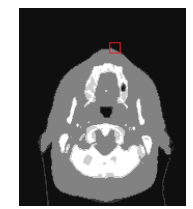

(c)

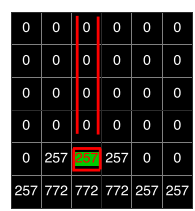

(d)
Fig. 3. (a) CT image; (b) Pixels of (a); (c) The segmented image after FODPSO; (d) Pixels of (c); different value is highlighted in a red square. Part-B

The steps described in part- $A$ will lead, in most cases, to correctly identifying pixels of the mask itself but it also misclassifies
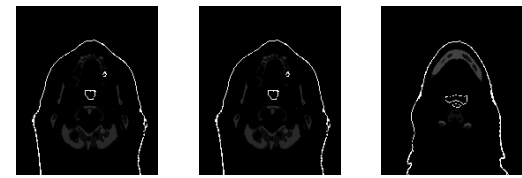

Fig. 4. Examples of segmented images produced after applying part$A$. The pixels that are identified as belonging to the immobilisation mask are displayed as brightly coloured. (Adjust Contrast Matlab Image Tool is used).
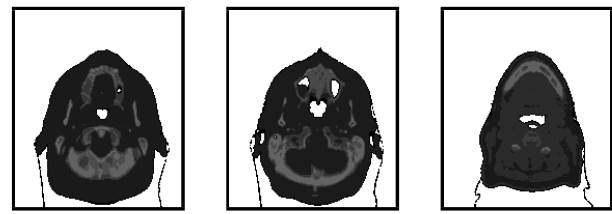

Fig. 5. Examples of segmented images produced after applying part- $A$. The pixels that are identified as belonging to the background were displayed in white. Note: Adjust Contrast Matlab Image Tool is used.

a small number of pixels located inside the skull. We render these as brightly coloured in Figure 4. This is because the FODPSO segmentation process groups some pixels comprising mask with others having similar X-ray absorption located inside the skull as one cluster. A similar problem exists with regard to background clusters (see Figure 5).

It is clear from our experiments, illustrated in Figure 4 and Figure 5 that the pixels misclassified by part- $A$ are always located inside the skull. Part- $B$ corrects this problem by recovering the coordinates of those pixels that form the skull and excluding these from the group of pixels that are belonging to the immobilisation mask. The approach comprises a sequence of operations that split the original CT image to two clusters (i.e. foreground(head) and background(air)) using Otsu's method. Then it automatically floodfills holes that may appear inside the skull using the morphological reconstruction operator described in [15]. After that, the pipeline proceeds by performing an image processing operation named erosion [16]. This will guarantee that no one of the pixels that are really belonging to the mask will be excluded later and then the only pixels that will be excluded are those which are exist inside the skull. The indices of those pixels which represent the skull will be gotten from the eroded image. Those indices will be stored in $H$ as the final output.

\section{Part-C}

Equations (1) and (2) are applied in part- $C$ to exclude those pixels which are misclassified in part- $A$ by applying an intersection operation between $M$ and $H$ in (1) and between $B$ and $H$ in (2).

$$
\begin{gathered}
M^{\prime}=M-(M \cap H) \\
B^{\prime}=B-(B \cap H)
\end{gathered}
$$

$M$ represents the indices of those pixels which are identified as belonging to the immobilisation mask. $B$ represents the indices of those pixels which are identified as background pixels. $H$ represents the indices of those pixels which are identified, in part$B$, as the pixels which form the area of the skull. Applying the two equations will produce new indices $M^{\prime}$ and $B^{\prime}$ where $M^{\prime}$ represents the indices of those pixels that are belonging to the immobilisation mask and $B^{\prime}$ represents the indices of those pixels which form the background after excluding the indices of pixels 
that are exist inside the skull. The original CT image is then updated with the value of $V \_b a c k g r o u n d$ on locations $M^{\prime}$ and $B^{\prime}$ which means the pixels that represent the mask will be replaced by the value of $V \_$background without affecting the values of other pixels in the original CT image.

\section{RESUlts}

Figure 6(a) displays an example of one of the X-ray CT slices from the first dataset. A previous study [17] evaluated the use of Particle Swarm Optimisation (PSO) for medical image segmentation and demonstrated the the FODPSO algorithm delivered high accuracy, stability and speed. We found that segmenting the image to six different classes tends to lead to better results as this number classifies all or most of the pixels belonging to the mask as one class. The FODPSO algorithm delivers significant benefits in terms of execution speed over the brute-force approach (i.e. exhaustive search) which takes a very long time when the number of clusters equals six. Figure 6(b) displays the image after rendering with the corresponding pixel labels.

In Figure 6(c-e) we present the output that is generated by part$B$. The image was firstly segmented to two classes (foreground and background) using Otsu's method. It was then filled automatically and eroded. Part- $A$ and part- $B$ of the approach produced three data structures of indices $(M, B$, and $H)$ and those indices were used to form the final output image which is displayed in Figure 6(f). Finally, Figure 7 illustrates the result of applying the approach to a random selection of input X-ray CT slices.

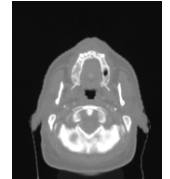

(a) CT image

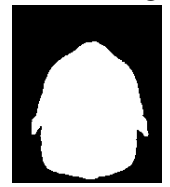

(d) Filled

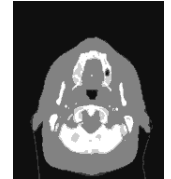

(b)(FODPSO)

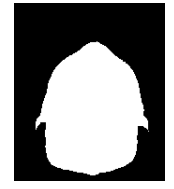

(e) Eroded

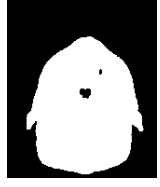

(c)(Otsu)

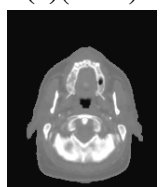

(f) Output
Fig. 6. An example of a CT slice from the first dataset.

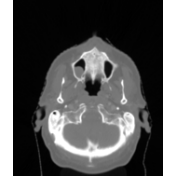

(a) In_D2

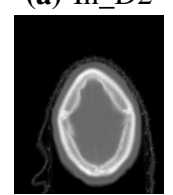

(e) In_D4

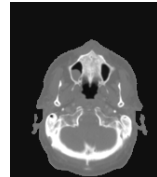

(b) Out_D2

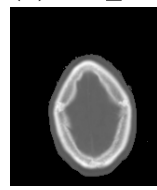

(f) out_D4

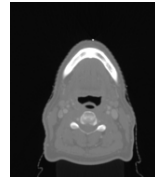

(c) In D3

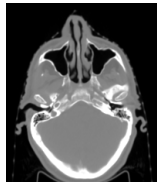

(g) In_D5

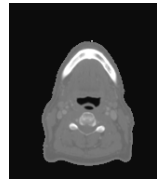

(d) Out_D3

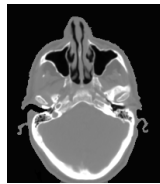

(h) Out_D5
Fig. 7. One CT slice example from each dataset (Input Output).

We used the Sensitivity and the Specificity to evaluate the proposed approach as both of them are statistical measures of the performance of a binary classification test. We have identified the True Positive (TP), False Positive (FP), True Negative (TN) and False Negative $(\mathrm{FN})$ in this context as:

TP: \# of mask pixels correctly identified as mask.

FP: \# of not-a-mask pixels incorrectly identified as mask.

TN: \# of not-a-mask pixels correctly identified as not-a-mask.

FN: \# of mask pixels incorrectly identified as not-a-mask.

The pixels that represent the immobilisation mask were identified by an expert in $25 \mathrm{CT}$ images (5 randomly-selected from each dataset) and compared to the number of pixels identified by the proposed approach. Table I displays the average values, rounded to the whole number, of TP, FP, TN and FN for each dataset and Table II displays the sensitivity, also called the true positive rate (TPR), specificity (SPC) and the Number-Of-Observations (NOO) for each dataset.

TABLE I

THE AVERAGE VALUES OF TP, FP, TN, AND FN FOR EACH DATASET

\begin{tabular}{lllll}
\hline Dataset & TP & TN & FP & FN \\
\hline Dataset\#1 & 389 & 30,239 & 100 & 23 \\
Dataset\#2 & 403 & 30,152 & 154 & 42 \\
Dataset\#3 & 429 & 30,199 & 93 & 30 \\
Dataset\#4 & 1714 & 29,060 & 465 & 203 \\
Dataset\#5 & 841 & 45,371 & 148 & 71 \\
\hline
\end{tabular}

TABLE II

THE VALUES OF TPR, SPC, AND NOO FOR EACH DATASET

\begin{tabular}{llll}
\hline Dataset & TPR & SPC & NOO \\
\hline Dataset\#1 & 0.9441 & 0.9967 & 30751 \\
Dataset\#2 & 0.9056 & 0.9949 & 30751 \\
Dataset\#3 & 0.9346 & 0.9969 & 30751 \\
Dataset\#4 & 0.8941 & 0.9842 & 31442 \\
Dataset\#5 & 0.9221 & 0.9967 & 46431 \\
Average & $\mathbf{0 . 9 2 0 1}$ & $\mathbf{0 . 9 9 3 9}$ & 34,025 \\
\hline
\end{tabular}

Table II shows the average value of the sensitivity (TPR) is $92.01 \%$ and the average value of the specificity (SPC) is $99.39 \%$. The heading 'NOO' in the table indicates to the number of observations which is equivalent to the number of pixels in each image.

\section{Increasing the speed of the approach}

Since HNC X-ray CT datasets normally comprises a large number of image slices, then applying a fast segmentation technique will play a key role in the speed of the proposed approach. We applied the brute-force method, PSO, Darwinian-PSO (DPSO) [17], and the FODPSO algorithm to segment the collection of CT slices under Otsu's criterion (i.e. maximising the inter-class variance between different clusters). The average time which is needed for each dataset to be segmented by each method is displayed in Table III. As shown in the table the FODPSO algorithm is the fastest and for that reason it makes it a good choice for our approach. We haven't recorded the time required by the brute-force method since it takes days for each dataset to be segmented using this method.

\section{Discussion}

We applied our approach over five different X-ray CT data sets, comprising a total of 738 image slices and noticed that the approach did not work on 13 of them $(<2 \%)$. Some of these represent cases 
where the heuristic search algorithm failed due to noise in the middle column, illustrated in Figure 8-left and some are cases in which the noise is so severe that the labels of true mask pixels is spatially discontinuous (see Figure 8-middle). We handled the first exception by applying the median filter over the background area in order to remove the noise from the background area, and we handled the second exception by changing the seeking mechanism in Algorithm 1 by searching the segmented image horizontally and vertically from different five start points, illustrated in Figure 8right.

Overall, we achieved an average specificity of $92.01 \%$ and sensitivity of $99.39 \%$ compared with ground truth provided by a human expert. Compared with other work segmenting therapy relevant anatomical structures in X-ray CT [7]-[10] these figures appear to be very promising and perhaps overly optimistic. However, we observe that the mask does not contact the skin at every pixel and there is sometimes a gap (quite desirable to achieve a comfortable fit) since masks are hand-made from plaster of paris moulds. We may speculate that for our application (i.e. not an embedded anatomical structure), automatic segmentation represents an easier task.
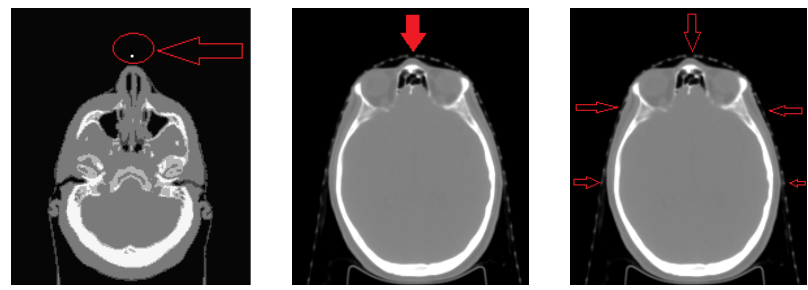

Fig. 8. (Left) Example of a CT image includes a noise in the middle column (Middle) Example of a CT image has a disconnected representation of the mask pixels (Right) Defining new start points to seek horizontally.

TABLE III

THE AVERAGE TIME (IN SEC) NEEDED FOR EACH DATASET TO BE SEGMENTED USING PSO, DPSO AND FODPSO ALGORITHMS

\begin{tabular}{lclll}
\hline Dataset & \# of images & PSO & DPSO & FODPSO \\
\hline Dataset\#1 & 155 & 130.99 & 107.18 & 103.90 \\
Dataset\#2 & 146 & 122.84 & 103.65 & 99.29 \\
Dataset\#3 & 151 & 126.96 & 102.82 & 100.99 \\
Dataset\#4 & 130 & 109.62 & 90.48 & 87.10 \\
Dataset\#5 & 156 & 131.41 & 109.84 & 107.08 \\
\hline
\end{tabular}

\section{CONCLUSiON}

This paper presented an automatic approach for identifying and removing artefacts due to immobilisation masks which normally exist in CT data sets of patients who are undergoing radiotherapy treatment for Head-and-Neck cancer. The presented approach accepts a CT image (in DICOM format) as an input, automatically identifies the pixels that belong to the immobilisation mask, sets the values of those pixels to be similar to the background value, thereby eliminating the mask from the output image. Five different data sets were tested to evaluate the accuracy of the approach. Sensitivity and specificity were used as statistical measures of the performance of the approach in this study. The evaluation indicates that the proposed approach is robust and of practical use. Some enhancements to speed up the process using PSO were also presented and tested in the paper.

\section{ACKNOWLEDGEMENT}

We would like to thank Prof. Susan Short and colleagues at St James's University Hospital NHS Foundation Trust for their help and for providing some of the data used in this study.

\section{REFERENCES}

[1] Head and Neck Cancer - Overview. http://www.cancer.net/ cancer-types/head-and-neck-cancer/overview. Accessed: 27-11-2016.

[2] The Office for National Statistics Report. https://www.ons.gov.uk/. Accessed: 27-11-2016.

[3] Ahmedin Jemal, Rebecca Siegel, Jiaquan Xu, and Elizabeth Ward. Cancer statistics, 2010. CA: a cancer journal for clinicians, 60(5):277-300, 2010.

[4] Head and Neck Cancer: Statistics. http://www.cancer.net/cancer-types/ head-and-neck-cancer/statistics. Accessed: 27-11-2016.

[5] Mark Fisher et al. Evaluation of 3-D printed immobilisation shells for head and neck IMRT. Open Journal of Radiology, 4(04):322, 2014.

[6] SD Laycock et al. Towards the production of radiotherapy treatment shells on 3D printers using data derived from dicom CT and MRI: preclinical feasibility studies. Journal of Radiotherapy in Practice, 14(01):92-98, 2015.

[7] Kaiping Wei, Bin He, Tao Zhang, and Xianjun Shen. A novel method for segmentation of CT head images. In 2007 1st International Conference on Bioinformatics and Biomedical Engineering, pages 717-720. IEEE, 2007.

[8] Wenan Chen, Rebecca Smith, Soo-Yeon Ji, and Kayvan Najarian. Automated segmentation of lateral ventricles in brain CT images. In Bioinformatics and Biomeidcine Workshops, 2008. BIBMW 2008. IEEE International Conference on, pages 48-55. IEEE, 2008.

[9] Xiaohua Qian, Jiahui Wang, Shuxu Guo, and Qiang Li. An active contour model for medical image segmentation with application to brain CT image. Medical physics, 40(2):021911, 2013.

[10] W Mimi Diyana W Zaki, M Faizal A Fauzi, R Besar, and WSH Munirah W Ahmad. Qualitative and quantitative comparisons of haemorrhage intracranial segmentation in CT brain images. In TENCON 2011-2011 IEEE Region 10 Conference, pages 369-373. IEEE, 2011.

[11] Nobuyuki Otsu. A threshold selection method from gray-level histograms. Automatica, 11(285-296):23-27, 1975.

[12] Kenneth Clark et al. The cancer imaging archive (TCIA): maintaining and operating a public information repository. Journal of digital imaging, 26(6):1045-1057, 2013.

[13] The Cancer Imaging Archive. http://doi.org/10.7937/K9/TCIA.2015. 7AKGJUPZ/. Bosch, Walter R. and Straube, William L. and Matthews, John W. and Purdy, James A. Data From Head Neck Cetuximab (2015).

[14] Micael S Couceiro, Rui P Rocha, NM Fonseca Ferreira, and JA Tenreiro Machado. Introducing the fractional-order darwinian pso. Signal, Image and Video Processing, 6(3):343-350, 2012.

[15] Soille Pierre. Morphological image analysis: Principles and applications, 1999.

[16] Rafael C Gonzalez and Richard E Woods. Image processing. Digital image processing, 2, 2007.

[17] Mohammad Hashem Ryalat et al. Evaluation of particle swarm optimisation for medical image segmentation. In Advances in Systems Science: Proceedings of the International Conference on Systems Science 2016 (ICSS 2016), pages 61-72. Springer, 2016. 